\title{
The concentration of ethanol affects its penetration rate in bovine cardiac and hepatic tissues
}

\author{
Matthew Dunster-Jones ${ }^{1}$, Michelle Steicke ${ }^{1}$, James Mackie', Rachel Guthrie ${ }^{1}$, \\ Tam Nguyen Dinh ${ }^{1}$, Fahima Ahmady ${ }^{1}$, Jonathan Golledge ${ }^{2,3}$, Yutang Wang ${ }^{1}$ \\ ${ }^{1}$ School of Applied and Biomedical Science, Federation University Australia, Ballarat, \\ Victoria, Australia \\ ${ }^{2}$ Vascular Biology Unit, Queensland Research Centre for Peripheral Vascular Disease, \\ College of Medicine and Dentistry, James Cook University, Townsville, Queensland, Australia \\ ${ }^{3}$ Department of Vascular and Endovascular Surgery, Townsville Hospital, Townsville, Australia
}

\begin{abstract}
Introduction. Ethanol is a commonly used fixative. Fixation of the inner layers of the tissue depends on the ability of the fixative to diffuse into the tissue. It is unknown whether the concentration of ethanol affects its penetration into tissues. This study aimed to compare the penetration rates of $50 \%$ and $100 \%$ ethanol into bovine heart and liver tissues.

Materials and methods. The penetration distance and tissue shrinkage or expansion were measured by analysing the digital images of the heart and liver tissues before and after immersion in ethanol at $20^{\circ} \mathrm{C}$ for $2,6,24$ or 30 hours. The penetration coefficients were calculated as the slope of the regression line using the linear regression function between the penetration distance and square root of fixation time. Differences in tissue shrinkage or expansion and penetration distance at various time points between the two concentrations of ethanol were analysed using a mixed design ANOVA followed by Bonferroni's post-hoc test.

Results. The penetration distance of $100 \%$ ethanol was significantly greater in both heart and liver tissues compared with that of $50 \%$ ethanol $(\mathrm{n}=4, \mathrm{p}<0.05$ for both). $100 \%$ ethanol shrank immersed liver tissue significantly more than $50 \%$ ethanol $(\mathrm{p}=0.002)$, but the shrinkage of the heart tissue caused by two concentrations of ethanol did not significantly differ $(\mathrm{p}=0.054)$. The greater penetration distance of $100 \%$ over $50 \%$ ethanol remained unchanged after normalising the penetration distance to the individual tissue's shrinkage $(\mathrm{n}=4$, $\mathrm{p}<0.001$ ). The mean penetration coefficient of $100 \%$ ethanol was significantly greater than $50 \%$ ethanol in the heart tissue $(0.906$ vs. $0.442, \mathrm{p}=0.003)$ and in the liver tissue $(0.988 v s .0 .622, \mathrm{p}=0.028)$.

Conclusions. It was proven that in two types of tissue that substantially differ in histological structures, $100 \%$ ethanol penetrated tissue significantly faster than 50\% ethanol. (Folia Histochemica et Cytobiologica 2018, Vol. 56, No. 2, 92-97)
\end{abstract}

Key words: fixative; ethanol; concentration; penetration coefficient; penetration rate

Correspondence address: Y. Wang

School of Applied and Biomedical Science,

Federation University Australia,

Ballarat, Victoria, 3353, Australia

tel.: +61 35327 9394; fax: +61353279289

e-mail: Yutang.wang@federation.edu.au

\section{Introduction}

Fixation is a critical step in the preparation of histological sections [1]. Ethanol is a commonly used fixative and it can be used alone $[2,3]$ or in combination with other fixatives [2, 4-6]. Fixation of the inner layers of tissues depends on the ability of the fixative to diffuse into the tissues. Traditionally it has been assumed that the penetration rate of a fixative is not affected by its concentration [7], but there has been no evidence reported to support this assumption. The aim of this 
study was to compare the penetration rates of $50 \%$ and $100 \%$ ethanol into bovine heart and liver tissues.

\section{Materials and methods}

Tissue fixation. Four fresh bovine hearts and four fresh bovine livers were purchased from a local butcher (Sinclair Meats). Hearts and livers were cut into $2 \times 2 \times 2 \mathrm{~cm}^{3}$ cubes ( 8 cubes from each heart or each liver). The top face of the tissue cube was marked with tissue dye. The tissue cubes were then immersed into $40 \mathrm{~mL}$ of fixatives $(50 \%$ or $100 \%$ ethanol) in a $50 \mathrm{~mL}$ specimen collection container and were kept in a room with the temperature controlled at $20^{\circ} \mathrm{C}$. During the course of fixation, the tissue cubes were immersed in the two concentrations of ethanol with the marked face always facing up.

Penetration distance measurement. The heart and liver cubes were taken out of the fixative at $2,6,24$ or $30 \mathrm{~h}$, and the marked face was photographed using a Sony Xperia Mobile camera (Sony, Tokyo, Japan) alongside a ruler. The tissue was then cut into two halves along the middle plane parallel to the marked face. The freshly cut face was photographed alongside a ruler for the penetration distance measurement. The discoloration area represented the penetrated area [2] (Fig. 1A). The penetration distance was measured 40 times at locations equally distributed around the perimeter of the cut face using PhotoShop software version CC 2017 (Adobe Systems Inc., San Jose, CA, USA). The mean of the 40 measurements was calculated as the penetration distance for an individual image.

Tissue shrinkage or expansion. The marked face of the tissue was photographed immediately before fixation (Fig. 2A) and 2, 6, 24 or $30 \mathrm{~h}$ after fixation (Fig. 2B). The area of the marked face of the tissue was measured using Photoshop software. The change in the marked surface area $(\Delta$ area $)$ was then calculated. The square root of the absolute value of $\Delta$ area was then calculated. The square root of the absolute value of the ratio of $\Delta$ area/area before fixation was then calculated as the shrinkage or expansion of the tissue. Negative values represented tissue expansion and positive values represented shrinkage.

Penetration coefficient. It has been observed that the penetration distance in millimetres $(x)$ of a given fixative depends on the following simple function of the fixation time in hours $(t)[3,8,9]$, i.e. $x=\mathrm{K} \vee \mathrm{t}$. The constant $\mathrm{K}$ is called the penetration coefficient. We plotted the penetration distance in millimetres relative to the square root of the fixation time in hours and obtained the penetration coefficient as the slope of the regression line using the linear regression function using IBM SPSS Statistics Software (Version 24.0, IBM Corp, New York, NY, USA). Coefficient of determi-

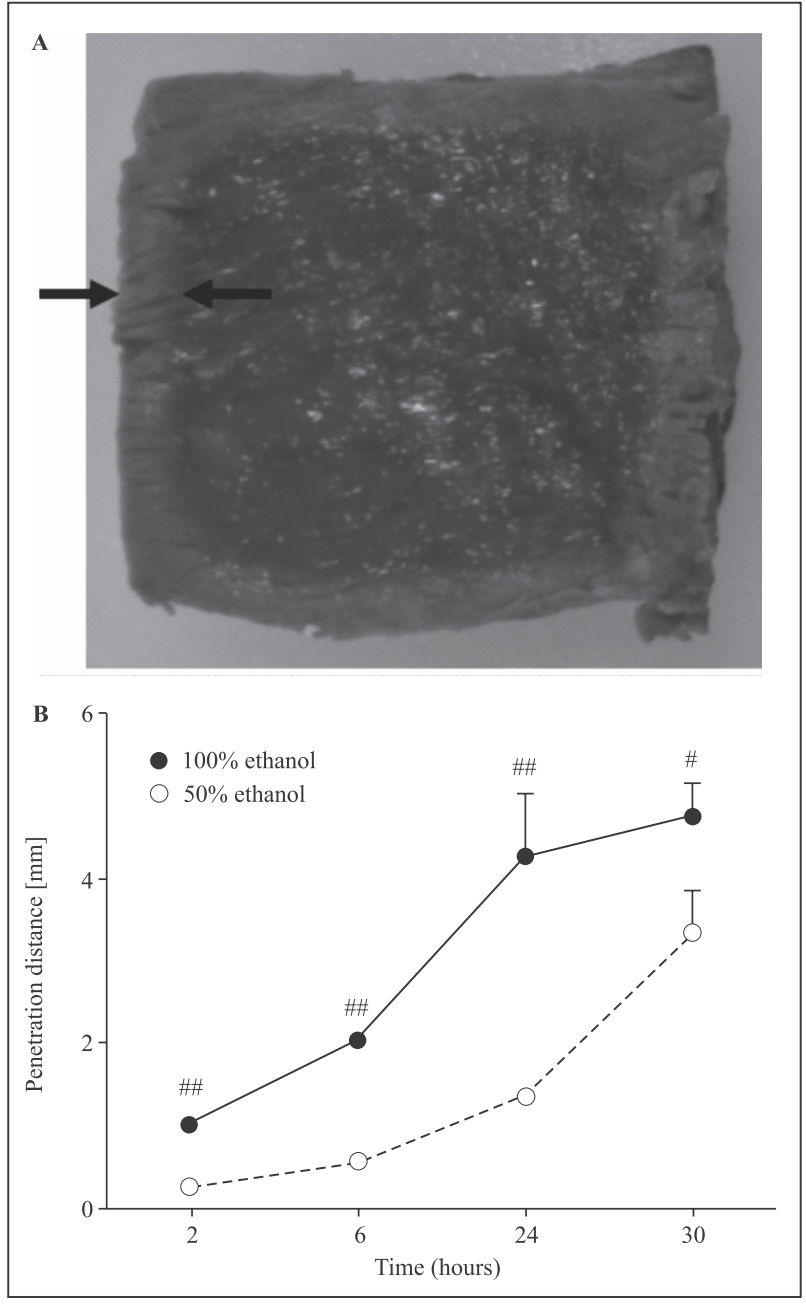

Figure 1. The penetration distance of ethanol in heart tissue. A. Representative image for the measurement of penetration distance. The penetration distance (the distance of discoloured region) was indicated between the two arrows, and it was measured 40 times at locations equally distributed around the perimeter. The mean of the 40 measurements was calculated as the penetration distance of the fixative in this sample. B. Penetration distance over time for $50 \%$ or $100 \%$ ethanol. ${ }^{\#} \mathrm{p}<0.01$ and ${ }^{\# \#} \mathrm{p}<0.001$ compared to $50 \%$ ethanol at the same time point, $n=4$. Error bars represent standard deviation.

nation $\left(\mathrm{R}^{2}\right)$ and $\mathrm{P}$ value were also obtained using the linear regression analysis.

Statistical analysis. Error bars in the figures represent standard deviation. Differences in shrinkage or expansion, penetration distance and penetration coefficient at various time points between the two concentrations of fixatives were analysed using a mixed design ANOVA using IBM SPSS Statistics Software (IBM), followed by Bonferroni's post-hoc test. The difference in penetration at various 


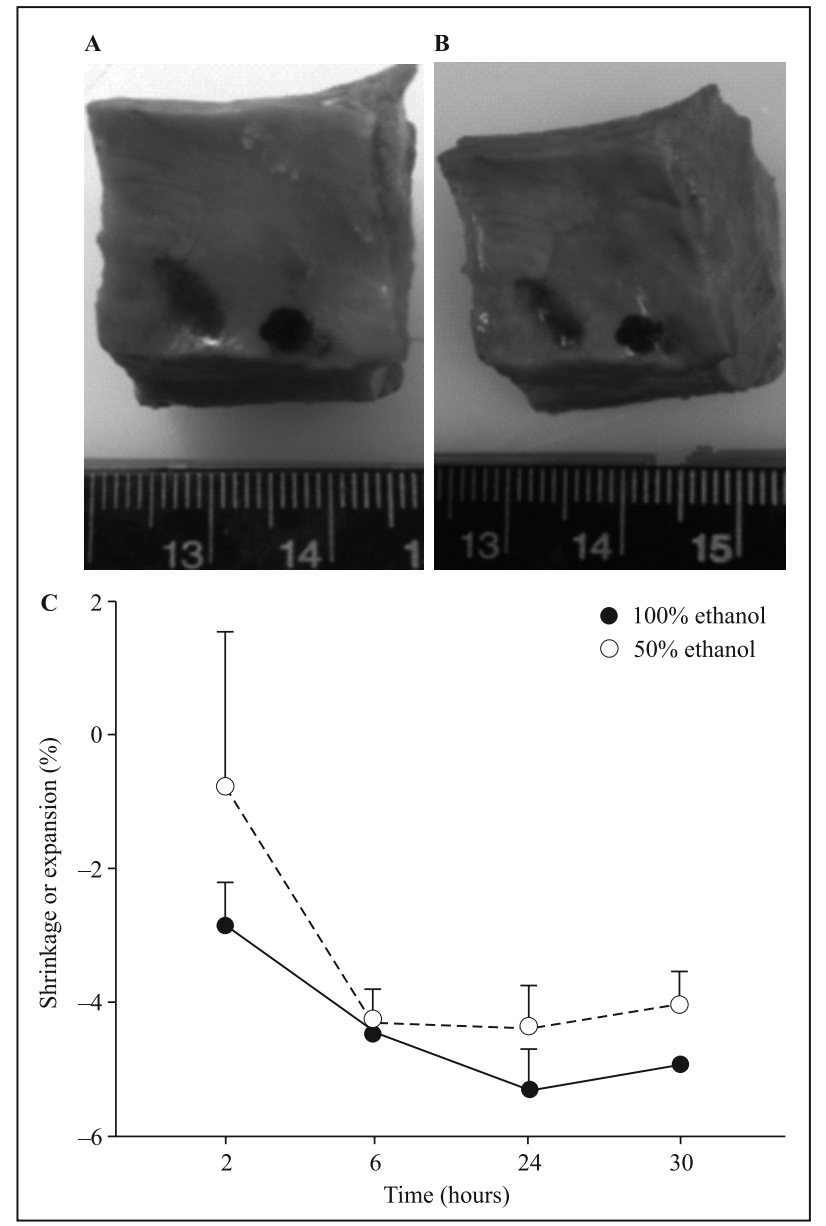

Figure 2. The tissue shrinkage or expansion induced by ethanol fixation. A. A typical image of a heart tissue cube before fixation. The tissue was marked on the top face for orientation, and the marked face was kept facing up during the course of the fixation. B. The image of the tissue cube after fixation. The tissue cube was the same as shown in image A. C. The shrinkage or expansion of the heart tissue after exposure to $50 \%$ or $100 \%$ ethanol for $2,6,24$ or $30 \mathrm{~h}$ at $20^{\circ} \mathrm{C}$. The change in the marked surface area ( $\Delta$ area) was calculated. The square root of the absolute value of the ratio of $\Delta$ area/area before fixation was then calculated as the shrinkage or expansion of the tissue. Positive values represent tissue expansion, whereas negative values represent tissue shrinkage; $n=4$.

time points for an individual fixative was analysed using one-way ANOVA.

\section{Results}

\section{The penetration rate of ethanol in the bovine heart}

First, we determined the variability in measuring penetration distance through three observers independently measuring the same images on four separate occasions. During each occasion penetration distance was assessed by 40 measurements at locations equally distributed around the perimeter of the tissue. The intra-observer coefficients of variation for the measurement of penetration distance by the three independent observers were $7.67 \%, 2.72 \%$ and $0.89 \%$, respectively (Supplementary Table 1 ). The inter-observer coefficient of variation was $3.56 \%$ (Supplementary Table 2).

The penetration distance in the heart tissue increased along with the time of fixation for both concentrations of ethanol ( $p<0.001$ for both, Fig. 1). The penetration distance of $100 \%$ ethanol in the heart tissue was significantly greater than that of $50 \%$ ethanol ( $p<0.001$, Fig. 1$)$.

Fixation can cause tissue shrinkage or expansion. Results of the effect of fixatives on penetration distance required normalisation of the individual tissue shrinkage or expansion. To measure the tissue shrinkage or expansion caused by ethanol, we compared the area of the marked face of the tissue cube before and after fixation. We determined the variability in measuring the area of the marked face by three observers independently measuring the marked face area of the same tissue cube 10 times. The intra-observer coefficients of variation of the three independent observers were $0.6 \%, 0.6 \%$ and $0.5 \%$, respectively (Supplementary Table 3), and the inter-observer coefficient of variation was $1.2 \%$ (Supplementary Table 4 ).

The heart tissue shrank at both ethanol concentrations (Fig. 2). The shrinkage caused by $100 \%$ ethanol was similar to that caused by $50 \%$ ethanol $(\mathrm{p}=0.054)$. After normalising the penetration distance to the individual tissue's shrinkage, the penetration distance of $100 \%$ ethanol was significantly greater than that of $50 \%$ ethanol ( $p<0.001$, Fig. 3A). The normalised penetration distance in millimetres was plotted relative to the square root of the fixation time in hours (Fig. 3B). The penetration distance was significantly correlated with the square root of the fixation time for both concentrations of ethanol (Table 1). The mean penetration coefficients $(\mathrm{K})$ in the heart tissue were 0.442 and 0.906 , for $50 \%$ and $100 \%$ ethanol, respectively (Table 1). The penetration coefficient of $100 \%$ ethanol was significantly greater than that of $50 \%$ ethanol $(\mathrm{p}=0.003$, Fig. $3 \mathrm{~B})$.

\section{The penetration rate of ethanol in the bovine liver}

The results obtained for the liver tissue were similar to those for the heart tissue. The penetration distance in the liver increased along with time of incubation for both concentrations of ethanol ( $p<0.001$ for both, Fig. 4A). The penetration distance of $100 \%$ ethanol in the liver was significantly greater than that of $50 \%$ ethanol ( $p=0.002$, Fig. 4A). The liver tissue shrank at both ethanol concentrations (Fig. 4B). The shrinkage 


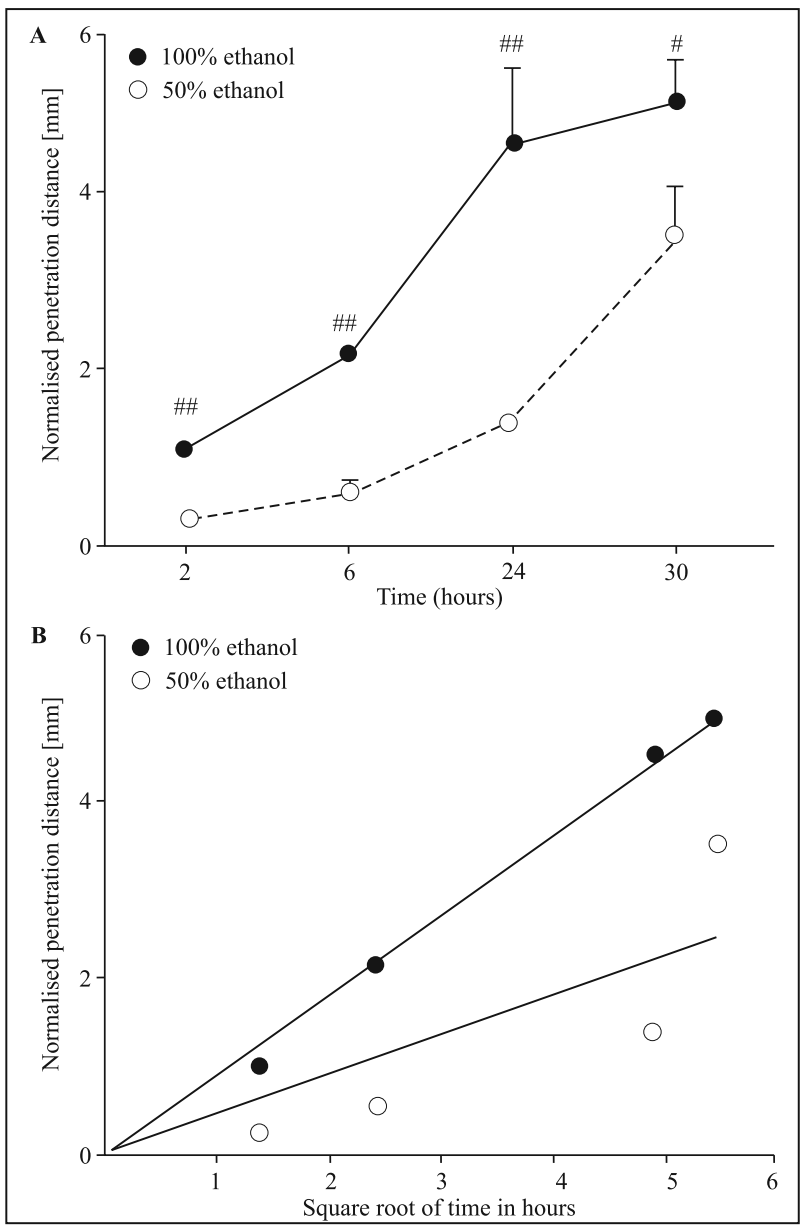

Figure 3. The normalised penetration distance of ethanol in heart tissue. A. The penetration distance was normalised to the tissue shrinkage or expansion of each individual tissue cube; $\mathrm{n}=4 ;{ }^{\#} \mathrm{p}<0.01$ and ${ }^{\# \#} \mathrm{p}<0.001$ compared to $50 \%$ ethanol at the same time point. B. The normalised penetration distance relative to the square root of the fixation time. The penetration distance in millimetres was the mean of the measurements from four heart tissues.

caused by $100 \%$ ethanol was significantly greater than that caused by $50 \%$ ethanol ( $\mathrm{p}=0.002$, Fig. 4B). After normalising the penetration distance to the individual tissue's shrinkage, the penetration distance of $100 \%$ ethanol was significantly greater than that of $50 \%$ ethanol ( $p<0.001$, Fig. 4C).
The normalised penetration distance in millimetres was plotted relative to the square root of the fixation time in hours (Fig. 4D). The penetration distance was significantly correlated with the square root of the fixation time for both concentrations of ethanol (Fig. 4D, Table 1). The mean penetration coefficients $(\mathrm{K})$ in the heart tissue were 0.622 and 0.988 , for $50 \%$ and $100 \%$ ethanol, respectively (Table 1 ). The penetration coefficient of $100 \%$ ethanol was significantly greater than that of $50 \%$ ethanol ( $p=0.028$, Fig. 4D).

\section{Discussion}

We report, for the first time, that the penetration rate of $100 \%$ ethanol was significantly greater than that of $50 \%$ ethanol in bovine heart and liver tissue pieces. Fixation with $100 \%$ ethanol resulted in greater tissue shrinkage than that with $50 \%$ ethanol in the liver tissue but not in the heart tissue. The difference in the penetration rate between $100 \%$ and $50 \%$ ethanol remained after the correction for tissue shrinkage. These results suggest that the assumption recently reported in a histotechnology textbook that the concentration of fixatives does not affect penetration rate is incorrect [7].

Ten percent neutral-buffered formalin is the preferred preservative in tissue fixation [10]. However, formalin fixation has limitations. For example formalin is toxic, carcinogenic, and a poor preserver of nucleic acids with poor performance in immunohistochemistry for the visualisation of some antigens [11]. Ethanol is a frequently used tissue fixative [2, 3]. The combination of ethanol with other fixatives has advantage to preserve the integrity of nucleic acids $[2,4-6,12]$ while improving the visibility of antigens [6].

Many factors affect fixation, e.g. temperature, fixation time, tissue thickness, and ratio of fixative to tissue volume [7]. These factors were kept consistent in our study. The tissue pieces were kept at $20^{\circ} \mathrm{C}$. Penetration was examined at 2, 6, 24 and 30 hours after fixation. $2 \times 2 \times 2 \mathrm{~cm}^{3}$ tissue cubes were used. The fixative volume used was $40 \mathrm{~mL}$. Therefore, the ratio of fixative to tissue volume was 5:1 in our

Table 1. Ethanol penetration coefficient $(\mathrm{K})$ in bovine heart and liver tissue

\begin{tabular}{|l|c|c|c|c|c|c|}
\hline & \multicolumn{3}{|c|}{$\mathbf{5 0 \%}$ ethanol } & \multicolumn{3}{c|}{ 100\% ethanol } \\
\cline { 2 - 7 } & $\mathbf{K}$ & $\mathbf{R}^{2}$ & $\mathbf{P}$ & K (mean \pm SE) & $\mathbf{R}^{2}$ & P \\
\hline Heart & $0.442 \pm 0.062$ & 0.834 & $<0.001$ & $0.906 \pm 0.029$ & 0.985 & $<0.001$ \\
\hline Liver & $0.622 \pm 0.034$ & 0.982 & $<0.001$ & $0.988 \pm 0.045$ & 0.983 & $<0.001$ \\
\hline
\end{tabular}

Data are expressed as mean $\pm \mathrm{SE}$. K, penetration coefficient, $\mathrm{R}^{2}-$ coefficient of determination. The penetration distance in millimetres (x) of a given fixative is a function of the fixation time in hours $(\mathrm{t})$, i.e. $\mathrm{x}=\mathrm{K} \sqrt{\mathrm{t}}$. The constant $\mathrm{K}$ is called the penetration coefficient. 


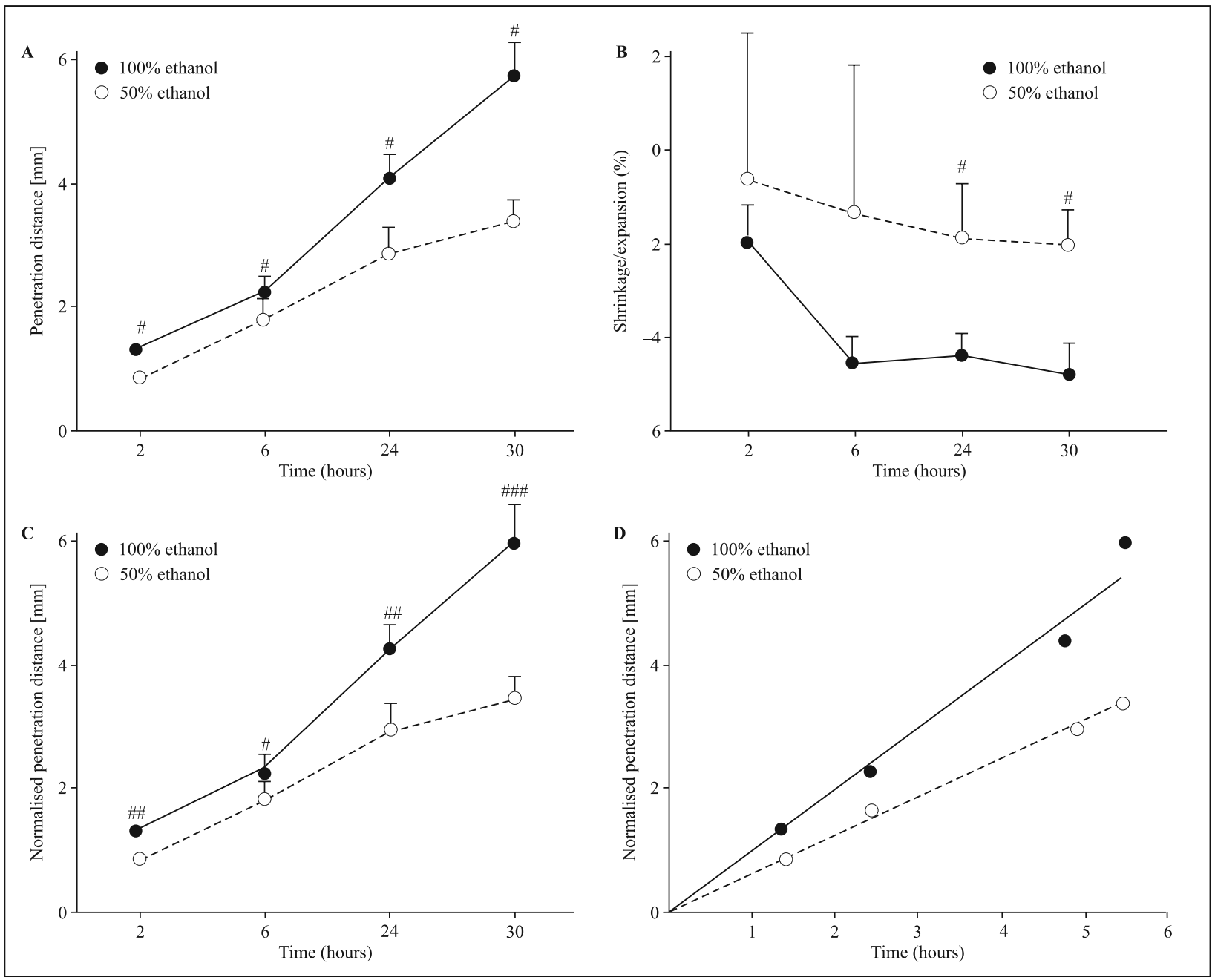

Figure 4. The penetration distance of different fixative concentrations in liver tissue. A. Penetration distance of $50 \%$ or $100 \%$ ethanol in the liver tissue for $2,6,24$ or $30 \mathrm{~h}$ at $20^{\circ} \mathrm{C} ; \mathrm{n}=4 ;{ }^{*} \mathrm{p}<0.01$ compared to $50 \%$ ethanol at the same time point. B. The shrinkage or expansion of the liver tissue after exposure to the fixatives for $2,6,24$ or $30 \mathrm{~h}$ at $20^{\circ} \mathrm{C}$. The change in the marked surface area ( $\Delta$ area) was calculated. The square root of the absolute value of the ratio of $\Delta$ area/area before fixation was then calculated as the shrinkage or expansion of the tissue. Positive values represent tissue expansion, whereas negative values represent tissue shrinkage; $\mathrm{n}=4 ;{ }^{\#} \mathrm{p}<0.01$ compared to $100 \%$ ethanol at the same time point. $\mathbf{C}$. The penetration distance in the liver tissue was normalised to the tissue shrinkage or expansion of each individual tissue cube; $\mathrm{n}=4$; ${ }^{\#} \mathrm{p}<0.05,{ }^{\# \#} \mathrm{p}<0.01$ and ${ }^{\# \# \#} \mathrm{p}<0.001$ compared to $50 \%$ ethanol at the same time point. D. The normalised penetration distance relative to the square root of the fixation time.

experiments. Researchers generally recommend that the ratio of fixative to tissue volume should be $10: 1$ or greater $[2,7]$. However, this recommendation lacks scientific evidence support. It has been reported that variation of fixative to tissue volume ratios (ranging from 1:1 to 20:1) did not affect fixation quality [13, 14]. Therefore, the fixative to tissue volume ratio of 5:1 in our study was appropriate.

When measuring the penetration distance of a fixative, Cabrera et al. only used 4 measurements to calculate the mean penetration distance [2]. To decrease the potential error, we measured the penetration distance 40 times at locations equally distributed around the perimeter of the freshly cut face. Our method had very good reproducibility with the intra-observer coefficients of variation of the penetration distance ranging from $0.89-7.67 \%$ and inter-observer coefficient of variation was $3.56 \%$.

The penetration coefficient of $100 \%$ ethanol was higher than that of $50 \%$ ethanol in both the bovine heart and liver tissue pieces providing the first evidence that the penetration rate of ethanol is affected by its concentration. Our results were consistent with other similar findings in the literature. For example, it was reported that when formalin concentrations increased from $8 \%$ to $9.5 \%$ and then to $40 \%$, the 
penetration coefficient increased from 5.875 to 6.117 and then to $6.722[8]$.

The penetration coefficient of ethanol in the liver was greater than that in the heart. The tissue-specific penetration rate has been previously reported. For example, the penetration of formalin into human skin was reported to be slower than that into human liver tissue [14]. The reason for the greater penetration rate into the bovine liver tissue than into the heart tissue in our study is not clear and this may be due to the fact the heart tissue has much denser consistency compared to the liver tissue which may hinder fixative diffusion.

The mean penetration coefficients of $100 \%$ ethanol in bovine heart and liver tissue pieces in our study were 0.906 and 0.988 at $20^{\circ} \mathrm{C}$, respectively. It has been reported that penetration coefficient of $100 \%$ ethanol was 1.714 in blood plasma coagulum at room temperature [8] and it was approximately 1.44 in rabbit livers at room temperature $\left(21-25^{\circ} \mathrm{C}\right)$ [3]. The difference among our study and these two literature reports may be due to the differences in temperature, tissue types and species.

Fixatives can cause tissue expansion or shrinkage [7]. To study the effect of fixatives on tissue expansion or shrinkage, we compared the area of the same face of the tissue cube before and after fixation. This method of measuring the area of a tissue face had good reproducibility, with the intra-observer coefficients of variation ranging from $0.5-0.6 \%$ and inter-observer coefficient of variation being $1.2 \%$. We found that both concentrations of ethanol shrank both heart and liver tissue pieces. This is consistent with previous reports $[2,7]$. Our results also showed that the degree of shrinkage caused by $100 \%$ ethanol was significantly greater than that caused by $50 \%$ ethanol in the liver tissue but not in the heart tissue. The tissue-specific effects of fixatives have been reported in previous studies. For example, formalin fixation expanded the porcine forelimb muscle tissue whereas it shrank the fat tissue [15].

\section{Conclusion}

This study showed that the penetration rate of $100 \%$ ethanol is significantly greater than that of $50 \%$ ethanol in both bovine heart and liver tissues. Our results suggest that the assumption that the penetration rate of a fixative is not affected by its concentration is incorrect. The concentration of a fixative needs to be considered when we search for alternative fixatives to replace hazardous ones.

\section{Conflict of interest}

The authors declare that they have no conflict of interest.

\section{Acknowledgements}

This work is funded by a grant from the National Health and Medical Research Council (1062671).

\section{References}

1. Evans D, Robinson M. What is histopatholgy. In: Orchard G, Nation B, editors. Histopathology. New York: Oxford University Press; 2012. p. PP 1-33.

2. Cabrera NC, Espinoza JR, Vargas-Jentzsch P, et al. Alcohol-based solutions for bovine testicular tissue fixation. J Vet Diagn Invest. 2017; 29(1): 91-99, doi: 10.1177/1040638716672252, indexed in Pubmed: 27852815.

3. Dempster Wt. Rates of penetration of fixing fluids. Am J Anat. 1960; 107: 59-72, doi: 10.1002/aja.1001070105, indexed in Pubmed: 13721811.

4. Harrison PT. An ethanol-acetic acid-formol saline fixative for routine use with special application to the fixation of non-perfused rat lung. Lab Anim. 1984; 18(4): 325-331, doi: 10.1258/002367784780865324, indexed in Pubmed: 6513498.

5. Mathieson W, Marcon N, Antunes L, et al. A Critical Evaluation of the PAXgene Tissue Fixation System: Morphology, Immunohistochemistry, Molecular Biology, and Proteomics. Am J Clin Pathol. 2016; 146(1): 25-40, doi: 10.1093/ajcp/ /aqw023, indexed in Pubmed: 27402607.

6. Staff S, Kujala P, Karhu R, et al. Preservation of nucleic acids and tissue morphology in paraffin-embedded clinical samples: comparison of five molecular fixatives. J Clin Pathol. 2013; 66(9): 807-810, doi: 10.1136/jclinpath-2012-201283, indexed in Pubmed: 23750036.

7. Carson FL, Hladik C. Histotechnology. 4th ed. Chicago: American Society for Clinical Pathology. ; 2014.

8. Medawar PB. - The rate of penetration of fixatives. J R Microsc Soc. 1941;61(1-2. : 46-57.

9. Start RD, Layton CM, Cross SS, et al. Reassessment of the rate of fixative diffusion. J Clin Pathol. 1992; 45(12): 1120-1121, indexed in Pubmed: 1479044.

10. Srinivasan M, Sedmak D, Jewell S. Effect of fixatives and tissue processing on the content and integrity of nucleic acids. Am J Pathol. 2002; 161(6): 1961-1971, doi: 10.1016/S00029440(10)64472-0, indexed in Pubmed: 12466110.

11. Buesa RJ. Histology without formalin? Ann Diagn Pathol. 2008; 12(6): 387-396, doi: 10.1016/j.anndiagpath.2008.07.004, indexed in Pubmed: 18995201.

12. Alshammari MA, Alshammari TK, Laezza F. Improved Methods for Fluorescence Microscopy Detection of Macromolecules at the Axon Initial Segment. Front Cell Neurosci. 2016; 10: 5, doi: 10.3389/fncel.2016.00005, indexed in Pubmed: 26909021.

13. Williams JH, Mepham BL, Wright DH. Tissue preparation for immunocytochemistry. J Clin Pathol. 1997; 50(5): 422-428, indexed in Pubmed: 9215127.

14. Buesa RJ, Peshkov MV. How much formalin is enough to fix tissues? Ann Diagn Pathol. 2012; 16(3): 202-209, doi: 10.1016/j.anndiagpath.2011.12.003, indexed in Pubmed: 22483550.

15. Docquier PL, Paul L, Cartiaux O, et al. Formalin fixation could interfere with the clinical assessment of the tumor-free margin in tumor surgery: magnetic resonance imaging-based study. Oncology. 2010; 78(2): 115-124, doi: 10.1159/000306140, indexed in Pubmed: 20357519. 